\title{
Crop Duster Jerk-off: a poetry of abuse
}

The crop duster jerk-off is strafing just over the rise, dropping clouds of fallout over "green light" wheat; he prides himself on his skill in tossing the plane about: small, but not insect-likein spite of the warning-sign paint-job: red and yellow stripes-it's a plane, just a plane tanked up with chemicals. He touts its fragility with an incessant buzz, a throttling up that draws attention: flicking about, if it comes down it will take us all out. This isn't "terrorism"? There's a strong wind blowing in our direction.

The baby is out of the house, for which I am grateful, but it will come back to an invisible coating.

A new layer to our occupation. They kill to make us grow, to feed the population. There's a chain of profit as perpetual and cyclical as a teething ring. Yelling into the tinctured air makes you hoarse, and the pilot gets off on it: it's in the loins, like reproduction, through sperm count dropping and the wheat changing colour. 